\title{
THE AGE-METALLICITY RELATION OF THE UNIVERSE IN NEUTRAL GAS: THE FIRST 100 DAMPED Ly $\alpha$ SYSTEMS
}

\author{
Jason X. Prochaska, ${ }^{1,2}$ Eric Gawiser, ${ }^{1,3,4,5}$ Arthur M. Wolfe, ${ }^{1,3}$ Sandra Castro, ${ }^{1,6,7}$ And S. G. Djorgovski ${ }^{1,6}$ \\ Received 2003 May 7; accepted 2003 August 8; published 2003 August 21
}

\begin{abstract}
We discuss accurate metallicity measurements for 125 damped Ly $\alpha$ (DLA) systems at $0.5<z<5$, including $\approx 50$ new measurements from our recently published Echellette Spectrograph and Imager surveys. This data set is analyzed to determine the age-metallicity relation of neutral gas in the universe. Contrary to previous analyses, this sample shows statistically significant evolution in the cosmic mean metallicity. The best linear fit for metallicity evolution is $-0.26 \pm 0.07$ dex per unit redshift. The DLA systems continue to maintain a floor in metallicity of $\approx 1 / 700$ solar, independent of observational effects. This metallicity threshold limits the prevalence of primordial gas in high-redshift galaxies and stresses the correspondence between damped systems and star formation. Finally, we comment on an apparent "missing metals problem:" the mean metallicity of the damped systems is $\approx 10$ times lower than the value expected from their inferred star formation history. This problem is evident in current theoretical treatments of galaxy formation and may indicate a serious flaw in our understanding of the interplay between star formation and metal enrichment.
\end{abstract}

Subject headings: galaxies: abundances — galaxies: ISM - quasars: absorption lines

On-line material: machine-readable table

\section{INTRODUCTION}

For the past decade, researchers have observed the damped Ly $\alpha$ (DLA) systems - quasar absorption-line systems with an $\mathrm{H}$ I column density $N(\mathrm{H} \mathrm{I}) \geq 2 \times 10^{20} \mathrm{~cm}^{-2}$-in order to trace cosmological properties of neutral gas in the early universe. With moderate-resolution spectroscopy, observers have taken a census of the H I mass density $\Omega_{\text {gas }}$ from $z=0$ to 5 and found DLA systems to comprise the majority of neutral gas out to at least $z=4$ (Lanzetta, Wolfe, \& Turnshek 1995; Wolfe et al. 1995; Storrie-Lombardi \& Wolfe 2000; Rao \& Turnshek 2000; Péroux et al. 2001; S. G. Djorgovski et al. 2003, in preparation). By combining these observations with higher resolution spectroscopy of metal-line transitions, one can track the metal enrichment of the universe in neutral gas (Pettini et al. 1994, 1997; Prochaska $\&$ Wolfe 2000). If the individual DLA metallicity measurements are weighted by their corresponding $\mathrm{H}$ I column densities, the resulting mean represents a cosmological quantity: $\Omega_{\text {metals }} / \Omega_{\text {gas }}$, which equals the mass-weighted metallicity $\langle Z\rangle$ of neutral gas in the universe (Lanzetta et al. 1995). Aside from selection biases, this statistic is independent of any physical property of the damped systems surveyed (e.g., mass and morphology). Therefore, it presents a fundamental test for theories of chemical evolution (e.g., Pei \& Fall 1995), and these observations constrain the star formation history of the universe and describe the in-

\footnotetext{
${ }^{1}$ Visiting Astronomer, W. M. Keck Telescope. The Keck Observatory is a joint facility of the University of California, the California Institute of Technology, and NASA.

${ }^{2}$ University of California Observatories, Lick Observatory, Natural Sciences II Annex, UC Santa Cruz, Santa Cruz CA 95064.

${ }^{3}$ Department of Physics, Code 0354, and Center for Astrophysics and Space Sciences, Code 0424, University of California at San Diego, 9500 Gilman Drive, La Jolla, CA 92093-0424.

${ }^{4}$ NSF Postdoctoral Fellow, Yale University, P.O. Box 208101, New Haven, CT 06520.

${ }^{5}$ Andes Prize Fellow, Universidad de Chile, Casilla 36-D, Santiago, Chile.

${ }^{6}$ Palomar Observatory, MS 105-24, California Institute of Technology, Pasadena, CA 91125.

${ }^{7}$ Infrared Processing and Analysis Center, MS 100-22, California Institute of Technology, Pasadena, CA 91125.
}

terplay between nucleosynthesis and gas enrichment in highredshift galaxies.

Previous investigations of $\langle Z\rangle$ showed no statistically significant evolution (Pettini et al. 1997, 1999; Prochaska \& Wolfe 2000; Prochaska \& Wolfe 2002, hereafter PW02) or only the hint of an increase at $z<1.5$ (Kulkarni \& Fall 2002). This lack of evolution challenged the models of chemical evolution and raised concerns that selection biases were limiting DLA surveys to an unrepresentative population of galaxies (e.g., Pettini et al. 1999). Because $\langle Z\rangle$ is an $N(\mathrm{H} \mathrm{I})$-weighted measure, the uncertainty in this statistic is dominated by the damped systems with the highest product of metallicity and $\mathrm{H}$ I column density. This is analogous to measurements of $\Omega_{\text {gas }}$ in which damped systems with the largest $N(\mathrm{H} \mathrm{I})$ dominate the uncertainty. ${ }^{8}$ For this reason, previous metallicity samples were susceptible to severe sample variance, systematic error, and potential outliers.

With the goal of reducing the effect of small sample size, we initiated a program (Prochaska, Gawiser, \& Wolfe 2001a) with the Echellette Spectrograph and Imager (ESI; Sheinis et al. 2002) to rapidly increase the sample of high- $z$ damped systems with accurate metallicity measurements. This instrument and our observing strategy have led to an increase in efficiency of nearly 10 times that of previous echelle observations. In this Letter, we report the principal results on chemical evolution from our ESI surveys of the DLA systems (Prochaska, Castro, \& Djorgovski 2003a; Prochaska et al. 2003b). Combining these new measurements with $\approx 75$ damped systems drawn from the literature, our analysis includes a sample of 125 DLA systems from $z=0.5$ to 5 .

\section{THE SAMPLE}

At present, over 300 DLA systems have been identified in the literature (Curran et al. 2002). Of these, approximately half provide a metallicity estimate. Unfortunately, these metallicity measurements are derived from a variety of telescopes with a range of instrumentation and therefore form a heterogeneous

\footnotetext{
${ }^{8}$ This effect is less severe at $z>4$ (see Péroux et al. 2001)
} 
TABLE 1

SUMMARY

\begin{tabular}{cccccccc}
\hline \hline QSO & $z_{\text {abs }}$ & $N(\mathrm{H} \mathrm{I})$ & $f_{[\mathrm{M} / \mathrm{H}]}^{\mathrm{a}}$ & {$[\mathrm{M} / \mathrm{H}]$} & $f_{[\mathrm{Fe} / \mathrm{H}]}^{\mathrm{b}}$ & {$[\mathrm{Fe} / \mathrm{H}]$} & $\mathrm{Refs}$. \\
\hline $\mathrm{Q} 0235+1615 \ldots \ldots$ & 0.526 & $21.80_{-0.100}^{+0.100}$ & 2 & $-0.22 \pm 0.15$ & 0 & $0.00 \pm 0.00$ & 1 \\
Q1622+238 $\ldots \ldots .$. & 0.656 & $20.36_{-0.100}^{+0.100}$ & 4 & $-0.87 \pm 0.25$ & 1 & $-1.27 \pm 0.15$ & 3,4 \\
Q1122-168 $\ldots \ldots .$. & 0.682 & $20.45_{-0.050}^{+0.050}$ & 4 & $-1.00 \pm 0.15$ & 1 & $-1.40 \pm 0.05$ & 5 \\
\hline
\end{tabular}

Note.-Table 1 is published in its entirety in the electronic edition of the Astrophysical Journal. A portion is shown here for guidance regarding its form and content.

References. - (1) V. T. Junkkarinen et al. 2003, in preparation; (3) Churchill et al. 2000; (4) Rao \& Turnshek 2000; (5) Ledoux, Bergeron, \& Petitjean 2002a.

${ }^{a} 1=\mathrm{Si}, \mathrm{S}$, or $\mathrm{O} ; 2=\mathrm{Zn} ; 3=\alpha$-element $+\mathrm{Zn}$ limits; $4=\mathrm{Fe}+0.4 ; 5=\mathrm{Fe}$ limits +0.4

${ }^{\text {b }} 1=\mathrm{Fe} ; 2,3=\mathrm{Fe}$ upper/lower limit; $4=\mathrm{Ni}-0.1 ; 5=\mathrm{Cr}-0.2 ; 6=\mathrm{Al} ; 11-16=$ limits.

sample of data (e.g., varying resolution, wavelength coverage, and signal-to-noise ratio $[\mathrm{S} / \mathrm{N}])$. We primarily restrict our analysis to DLA systems observed on the current generation of large telescopes (e.g., Keck and the Very Large Telescope) with high-resolution $(R>5000)$, high-S/N $\left(15\right.$ pixel $\left.^{-1}\right)$ spectra. Data of poorer quality often have understated statistical errors and underappreciated systematic errors. We limit the analysis to systems satisfying the strict $N(\mathrm{H} \mathrm{I}) \geq 2 \times 10^{20} \mathrm{~cm}^{-2}$ DLA criterion. This facilitates comparisons with DLA surveys and simplifies comparisons with theoretical models.

Table 1 lists the name, $z_{\text {abs }}, N(\mathrm{H} \mathrm{I})$, metallicity $[\mathrm{M} / \mathrm{H}], \mathrm{Fe}$ abundance $[\mathrm{Fe} / \mathrm{H}]$, and reference for the 125 DLA systems comprising the sample. When possible, we adopt an $[\mathrm{M} / \mathrm{H}]$ value derived from an abundance measurement of an $\alpha$-element or $\mathrm{Zn}$ (with decreasing priority: $\mathrm{Si}, \mathrm{S}, \mathrm{O}, \mathrm{Zn}$ ). These elements are mildly refractory or nonrefractory and exhibit solar-relative abundances with few exceptions in the DLA system (PW02). In cases for which there are only limits reported to these abundances and the limits span an interval of less than 0.4 dex, we adopt the central value and an error encompassing the two limits. As a last resort (eight cases), we adopt an $[\mathrm{M} / \mathrm{H}]$ value calculated from $[\mathrm{Fe} / \mathrm{H}]$ assuming an offset of $0.4 \mathrm{dex}$. This offset matches the median and mean $[\alpha / \mathrm{Fe}]$ and $[\mathrm{Zn} / \mathrm{Fe}]$ values for DLA systems at $z>2$ (PW02). Figure 1 presents the full set of $[\mathrm{M} / \mathrm{H}]$ values as a

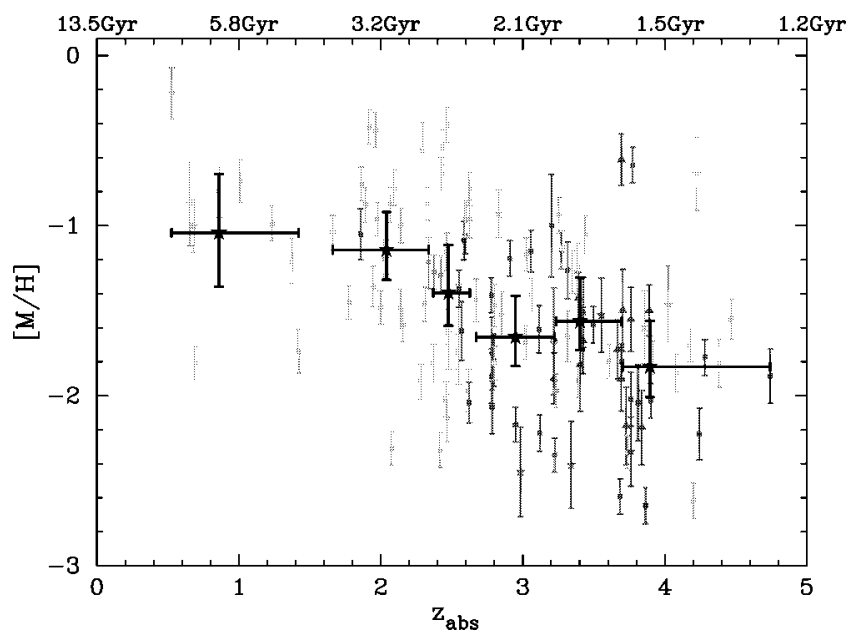

FIG. 1.-The $\left([\mathrm{M} / \mathrm{H}], z_{\text {abs }}\right)$-values for the 125 damped systems. The dark, unbinned points indicate the new values from our ESI surveys, while the lighter points are taken from the literature (primarily HIRES and UVES observations). The symbols indicate the origin of the $[\mathrm{M} / \mathrm{H}]$ values: $\alpha$-element measurement (filled squares); $\mathrm{Zn}$ measurement (filled circle); $\alpha+\mathrm{Zn}$ limits (filled triangle); Fe measurement +0.4 dex (filled star); Fe limits +0.4 dex (open circle). The unweighted mean logarithmic metallicity in the six bins is shown as a solid star with a $95 \%$ c.l. determined by our bootstrap technique. This statistic was chosen to best represent the evolution in the metallicity of a "typical" damped system as seen by eye in the plot. A least-squares fit to the center of the error intervals yields a best-fit slope of $m=-0.28 \pm 0.05 \mathrm{dex}$. function of $z_{\text {abs }}$. For most data points, the error is dominated by the uncertainty in $N(\mathrm{H} \mathrm{I})$ measurements. The quoted values tend to overestimate the statistical error and underestimate the systematic error from line blending and continuum placement. We adopt a minimum error of 0.1 dex for all metallicity measurements and note that none of our results are sensitive to uncertainty in the individual measurements.

\section{ANALYSIS}

In Figure 1, we overplot the unweighted, mean logarithmic metallicity in five redshift bins at $z>1.5$ defined to have equal numbers of DLA systems and one redshift bin with $z=$ $[0.5,1.5]$ covering the damped systems with $\operatorname{Ly} \alpha$ profiles at $\lambda_{\text {obs }}<3100 \AA$. The vertical error bars report a $95 \%$ confidence level (c.l.) uncertainty in this mean value as determined from a bootstrap error analysis. Meanwhile, the redshift marked for each bin refers to the median $z_{\text {abs }}$ value. An eyeball analysis of Figure 1 reveals evolution in the unweighted mean metallicity. Performing a least-squares linear fit to the binned data (accounting for the asymmetry in the error intervals), we calculate a slope $m=-0.28 \pm 0.05 \mathrm{dex} / \Delta z$ and a zero point $[\mathrm{M} / \mathrm{H}]_{0}=-0.67 \pm 0.16 \mathrm{dex}$. The results indicate that the metallicity of the "average" galaxy is increasing with decreasing redshift with an $e$-folding time of approximately 1 unit redshift, i.e., a decrease of 2 times per gigayear at $z \sim 3$. This evolution in the unweighted mean contrasts with our previous samples (e.g., PW02), although Vladilo et al. (2000) suggested such a trend at $z<3$ in a much smaller sample. We note that the $y$ intercept of the best-fit line is $4 \sigma$ below solar metallicity. This may be an indication of small sampling at low $z$ or a failure of the assumption that the logarithmic mean metallicity evolves linearly with redshift. On the other hand, a cross sectionselected sample of $\mathrm{H}$ I gas at $z=0$ (e.g., Rosenberg \& Schneider 2003) may show a subsolar metallicity because of the contributions from dwarf and low surface brightness galaxies.

The cosmological mean metallicity $\langle Z\rangle$ was computed using $\langle Z\rangle=\log \left[\sum_{i} 10^{[\mathrm{M} / \mathrm{H}]_{i}} N(\mathrm{H} \mathrm{I})_{i} / \sum_{i} N(\mathrm{H} \mathrm{I})_{i}\right]$ and is presented in Figure 2 in the same six bins. Because the $\langle Z\rangle$ statistic is dominated by the DLA system with the largest $N(\mathrm{H} \mathrm{I})$ and $[\mathrm{M} / \mathrm{H}]$ values, its measurement uncertainty will always be dominated by sample variance as opposed to statistical error. We estimate this variance through the bootstrap technique, and the vertical error bars refer to a $95 \%$ c.l. on the mean. The bootstrap technique assesses the sample variance under the assumption that the observed distribution is not severely different from the true distribution. If the data sample is too small, even a single outlier could significantly change the central value of the mean statistic beyond the uncertainty implied by the bootstrap analysis. While this issue was a concern in previous analyses, the results at $z \approx 2-4$ are now robust to all but the unlikeliest of 


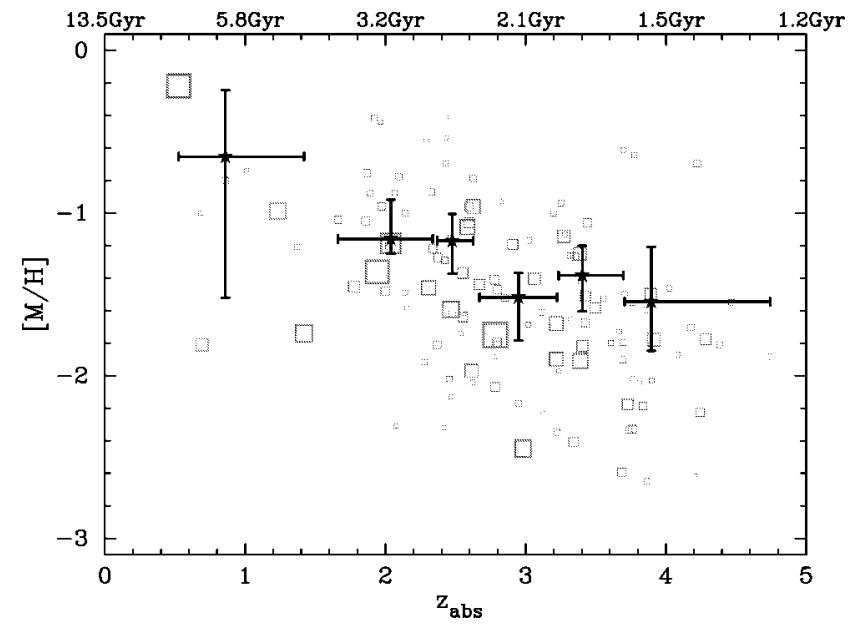

FIG. 2.-The $\left([\mathrm{M} / \mathrm{H}], z_{\text {abs }}\right)$-pairs plotted as open squares where the area of each square is scaled to the $N\left(\mathrm{HI}_{\mathrm{I}}\right)$ value of the DLA system. This aids the eye in determining which sight lines dominate the $N(\mathrm{H} \mathrm{I})$-weighted, cosmic mean metallicity. The cosmic metallicity $\langle Z\rangle$ is plotted for six bins with $95 \%$ c.l. uncertainties given by a bootstrap analysis. We find a best-fit slope of $m=-0.26 \pm 0.07$ dex, assuming a linear solution to the $\langle Z\rangle$ vs. $z_{\text {abs }}$ values.

outliers. This is primarily due to the large increase in total $N(\mathrm{H} \mathrm{I})$ sampled by the new observations. The ESI samples include 17 DLA systems with $N(\mathrm{H} \mathrm{I})>10^{21} \mathrm{~cm}^{-2}$, a $160 \%$ increase over previous samples. For example, the discovery of a system with $N(\mathrm{H} \mathrm{I})=10^{22} \mathrm{~cm}^{-2}$ and $\frac{1}{3}$ solar metallicity would increase $\langle Z\rangle$ by only $\approx+0.2 \mathrm{dex}$ in the $z \sim 2$ bin (see also Prochaska 2003). Such an outlier would lie nearly a factor of 10 off the current $N(\mathrm{H} \mathrm{I})-[\mathrm{M} / \mathrm{H}]$ distribution. It would represent a population of DLA systems that is rare and physically distinct from the 125 DLA systems presented here, unless the current sample is significantly biased by selection effects (e.g., dust obscuration), an assertion unsupported by any recent observational test (e.g., Ellison et al. 2001b; PW02).

Performing a least-squares fit to the $\langle Z\rangle$ values, we find $m=-0.26 \pm 0.07$ and $b=-0.59 \pm 0.18$ dex. ${ }^{9}$ Somewhat to our surprise, especially given the impression from a visual inspection of Figures 1 and 2, the best-fit slope to the $\langle Z\rangle$ values is identical (within statistical uncertainties) to the slope for the unweighted mean. This marks the first demonstration of evolution in $\langle Z\rangle$ at greater than $3 \sigma$ confidence. In this case, our analysis implies that the mean metallicity of the universe in neutral gas is roughly doubling every billion years at $z \approx 3$. We also note that dust depletion could imply a slightly steeper slope $(\approx 0.1 \mathrm{dex})$ than reported here, especially if $\mathrm{Si}$ is depleted in metal-rich DLA systems (Vladilo 2002).

\section{DISCUSSION}

The history of metallicity in the universe provides an important constraint on models of galaxy formation. A principal result from this study is that the DLA systems exhibit a modest, statistically significant evolution in metallicity with time. This conclusion contradicts earlier claims-including our own (e.g., PW02) — of no evolution, although it results almost entirely from the larger size of the current sample. Most importantly, the results reduce the likelihood that the DLA systems are a biased or transient population disconnected from general galactic evolution. This point is further emphasized by the nearly

\footnotetext{
${ }^{9}$ Note that the inclusion of the $z<1.5$ bin has minimal impact on this analysis.
}

constant scatter among the $[\mathrm{M} / \mathrm{H}]$ points with redshift. If the DLA systems consisted primarily of galaxies undergoing a transient phase or if they represented a continuously forming population of galaxies, one would expect no evolution in the mean metallicity with time and/or an increasing scatter in the distribution of $[\mathrm{M} / \mathrm{H}]$ values. Of course, these assertions are best investigated through detailed models of chemical evolution.

Another important characteristic of the observed distribution of $([\mathrm{M} / \mathrm{H}], z)$-pairs is the areas in redshift-metallicity space that remain unoccupied. In particular, note the absence of any DLA systems at $[\mathrm{M} / \mathrm{H}]<-3$ and the lack of DLA systems at $[\mathrm{M} / \mathrm{H}]>0$. Regarding the lower limit, it appears possible that we will never identify a DLA system with $[\mathrm{M} / \mathrm{H}]<-3$, a value that significantly exceeds our detection limit. This lower bound has important implications for the presence of primordial (zero metallicity) gas within these galaxies. If primordial gas with significant surface density and large cross section exists in highredshift galaxies, then it is always surrounded by metal-enriched gas yielding a mass-weighted metallicity exceeding 1/1000 solar. Alternatively, primordial gas may not exist in the neutral phase within high- $z$ galaxies. This metallicity floor also limits the contribution to the DLA population from gas "clouds" that are unrelated to galaxies (e.g., overdense regions associated with largescale structures). The metallicities of even the most metal-poor DLA systems $([\mathrm{M} / \mathrm{H}] \approx-2.6)$ are higher than the metallicities typically attributed to the Ly $\alpha$ forest (e.g., Songaila 2001). While the lower bound to the DLA values could be related to the physical processes that have enriched the Ly $\alpha$ forest (e.g., prompt enrichment from Population III stars; Wasserburg \& Qian 2000), this offset between the DLA and Ly $\alpha$ forest metallicities suggests that the enrichment of DLA gas is dominated by metal production from star formation within these galaxies. An alternative to this argument, however, is a Population III scenario that predicts significantly larger pre-enrichment in regions of higher overdensity (e.g., Schaye et al. 2003).

A key implication of our results comes to light when we compare them with the recent determination of DLA star formation rates (Wolfe, Prochaska, \& Gawiser 2003b). Although it is not yet possible to compare the metallicity and integrated star formation history of individual DLA systems, it is possible to compare the average DLA metallicity at $z=2.5$ with the integral under the DLA cosmic star formation history at $z>2.5$ (see Fig. 6 of Wolfe, Gawiser, \& Prochaska 2003a). The comparison illustrates a "missing metals problem" for the DLA systems that is similar to the problem described by Pettini (1999): the integrated star formation rates imply 10 times the mass density of metals observed in DLA systems. This conflict suggests scenarios in which the metals are ejected from the galaxy via supernova feedback (e.g., Mac Low \& Ferrara 1999) or in which the metals are sequestered within the star formation regions for significant timescales (e.g., within a galactic bulge; Wolfe et al. 2003a). Hence, it is possible that the mean metallicities of the typical DLA systems are not representative of those in the actual star-forming regions. Observations of absorption systems associated with gamma-ray bursts, which probably do probe the starforming regions themselves, suggest higher metallicities that may be more typical of disk stellar populations (see, e.g., Mirabal et al. 2002, Savaglio, Fall, \& Fiore 2003, Djorgovski et al. 2003, and references therein). A fundamental uncertainty in comparing the actively star-forming regions sampled by gamma-ray bursts and that of the majority of the neutral gas in the universe sampled by the DLA systems is our lack of understanding of the mixing efficiency of metal enrichment.

This problem is evident in recent theoretical models of chem- 
ical evolution within hierarchical galaxy formation scenarios (Somerville, Primack, \& Faber 2001; Tissera et al. 2001; Mathlin et al. 2001; Cen et al. 2003). The analyses appear to offer a wide range of theoretical values for the mean metallicity of the universe in neutral gas, yet the majority of variation stems from two key factors: (1) the adopted (or predicted) star formation history and (2) the treatment of selection biases due to dust obscuration. An appreciation of these two aspects can be obtained by following the analysis of Somerville et al. (2001), who focused on the stellar properties of the Lyman break galaxies. In all of their star formation scenarios, the $\langle Z\rangle$ values exceeded the DLA observations by at least a factor of 3 at all redshifts $z>$ 2. This discrepancy is a restatement of the "missing metals problem" described above. At present, the star formation history implied by various studies of high- $z$ galaxies (including DLA systems themselves) produces too many metals in comparison with metallicity observations of the DLA systems.

Several of the theoretical treatments, however, report successes in matching previous DLA metallicity samples. In all of these cases, the authors introduced significant selection biases owing to the effects of dust obscuration. Undoubtedly, dust obscuration plays a role in observations of the DLA systems, especially at lower redshift where the gas metallicity and dust content are presumably highest. At $z>2$, however, current samples of DLA systems toward radio-selected quasars exhibit no significant difference from optically selected samples (Ellison et al. 2001b). Furthermore, PW02 found no dependence between the inferred dust opacity of observed DLA sight lines and the quasar magnitude, contrary to expectations in scenarios for which dust obscuration is important. The original claims of reddening by Pei, Fall, \& Bechtold (1991) have not been confirmed by larger, homogeneous quasar samples; e.g., Outram et al. (2001) found only a $2 \sigma$ indication of reddening in their $z \lesssim 12 \mathrm{dF}$ quasar sample. At present, we see no compelling evidence of sample bias related to dust obscuration beyond its possible solution to the missing metals problem (e.g., Pei, Fall, \& Hauser 1999). We believe the mismatch between the star formation history of the early universe and the metallicity measurements of the DLA systems reflects our ignorance of a vital aspect of chemical enrichment that requires careful future investigation.

We acknowledge the Keck staff for their efforts in performing these observations. We thank M. Pettini for helpful comments and acknowledge collaborators S. Rao and D. Turnshek, who allowed us to present measurements prior to publication. E. G. is supported by NSF award AST 02-01667. A. M. W. is partially supported by NSF grant AST 00-71257. S. G. D. acknowledges partial support from the Ajax Foundation.

\section{REFERENCES}

Boissé, P., Le Brun, V., Bergeron, J., \& Deharveng, J.-M. 1998, A\&A, 333, 841 Cen, R., Ostriker, J. P., Prochaska, J. X., \& Wolfe, A. M. 2003, ApJ, submitted Churchill, C. W., Mellon, R. R., Charlton, J. C., Jannuzi, B. T., Kirhakos, S., Steidel, C. C., \& Schneider, D. P. 2000, ApJS, 130, 91

Curran, S. J., Webb, J. K., Murphy, M. T., Bandiera, R., Corbelli, E., \& Flambaum, V. V. 2002, Publ. Astron. Soc. Australia, 19, 455

Dessauges-Zavadsky, M., D’Odorico, S., McMahon, R. G., Molaro, P., Ledoux, C., Péroux, C., \& Storrie-Lombardi, L. J. 2001, A\&A, 370, 426

Djorgovski, S. G., et al. 2003, in Gamma-Ray Bursts in the Afterglow Era: Third Workshop, ed. L. Piro \& M. Feroci (San Francisco: ASP), in press (astro-ph/0302004)

Ellison, S. L., Pettini, M., Steidel, C. C., \& Shapely, A. E. 2001a, ApJ, 549, 770

Ellison, S. L., Yan, L., Hook, I. M., Pettini, M., Wall, J. V., \& Shaver, P. 2001b, A\&A, 379, 393

Kulkarni, V. P., \& Fall, S. M. 2002, ApJ, 580, 732

Lanzetta, K. M., Wolfe, A. M., \& Turnshek, D. A. 1995, ApJ, 440, 435

Ledoux, C., Bergeron, J., \& Petitjean, P. 2002a, A\&A, 385, 802

Ledoux, C., Petitjean, P., \& Srianand, R. 2003, A\&A, submitted (astro-ph/ 0302582)

Ledoux, C., Srianand, R., \& Petitjean, P. 2002b, A\&A, 392, 781

Lopez, S., \& Ellison, S. L. 2003, A\&A, 403, 573

Lopez, S., Reimers, D., D’Odorico, S., \& Prochaska, J. X. 2002, A\&A, 385, 778

Lopez, S., Reimers, D., Rauch, M., Sargent, W. L. W., \& Smette, A. 1999, ApJ, 513, 598

Lu, L., Sargent, W. L. W., \& Barlow, T. A. 1999, in Highly Redshifted Radio Lines, ed. C. L. Carilli, S. J. E. Radford, K. M. Menten, \& G. I. Langston (San Fransisco: BookCrafters Inc.), 132

Lu, L., Sargent, W. L. W., Barlow, T. A., Churchill, C. W., \& Vogt, S. 1996, ApJS, 107, 475

Mac Low, M.-M., \& Ferrara, A. 1999, ApJ, 513, 142

Mathlin, G. P., Baker, A. C., Churches, D. K., \& Edmunds, M. G. 2001, MNRAS, 321, 743

Meyer, D. M., Lanzetta, K. M., \& Wolfe, A. M. 1995, ApJ, 451, L13

Mirabal, N., et al. 2002, ApJ, 578, 818

Molaro, P., Bonifacio, P., Centurión, M., D’Odorico, S., Vladilo, G., Santin, P., \& Di Marcantonio, P. 2000, ApJ, 541, 54

Outram, P. J., Smith, R. J., Shanks, T., Boyle, B. J., Croom, S. M., Loaring,

N. S., \& Miller, L. 2001, MNRAS, 328, 805

Pei, Y. C., \& Fall, S. M. 1995, ApJ, 454, 69

Pei, Y. C., Fall, S. M., \& Bechtold, J. 1991, ApJ, 378, 6

Pei, Y. C., Fall, S. M., \& Hauser, M. G. 1999, ApJ, 522, 604

Péroux, C., Petitjean, P., Aracil, B., \& Srianand, R. 2002, NewA, 7, 577

Péroux, C., Storrie-Lombardi, L. J., McMahon, R. G., Irwin, M., \& Hook, I. M. 2001, AJ, 121, 1799

Petitjean, P., Srianand, R., \& Ledoux, C. 2002, MNRAS, 332, 383
Pettini, M. 1999, in Proc. ESO Workshop, Chemical Evolution from Zero to High Redshift, ed. J. R. Walsh \& M. R. Rosa (Berling: Springer), 233

Pettini, M., Ellison, S. L., Bergeron, J., \& Petitjean, P. 2002, A\&A, 391, 21

Pettini, M., Ellison, S. L., Steidel, C. C., \& Bowen, D. V. 1999, ApJ, 510, 576

Pettini, M., Ellison, S. L., Steidel, C. C., Shapely, A. L., \& Bowden, D. V. 2000, ApJ, 532, 65

Pettini, M., Lipman, K., \& Hunstead, R. W. 1995, ApJ, 451, 100

Pettini, M., Smith, L. J., Hunstead, R. W., \& King, D. L. 1994, ApJ, 426, 79

Pettini, M., Smith, L. J., King, D. L., \& Hunstead, R. W. 1997, ApJ, 486, 665

Prochaska, J. X. 2003, in Carnegie Symp.: Abundances, ed. A. McWilliam \& M. Rauch, in press

Prochaska, J. X., Castro, S., Djorgovski, S. G. 2003a, ApJS, in press

Prochaska, J. X., Gawiser, E., \& Wolfe, A. M. 2001a, ApJ, 552, 99

Prochaska, J. X., Gawiser, E., Wolfe, A. M., Cooke, J., \& Gelino, D. 2003b, ApJS, in press

Prochaska, J. X., Howk, J. C., O’Meara, J. M., Tytler, D., Wolfe, A. M., Kirkman, D., Lubin, D., \& Suzuki, N. 2002, ApJ, 571, 693

Prochaska, J. X., Howk, J. C., \& Wolfe, A. M. 2003c, Nature, in press

Prochaska, J. X., \& Wolfe, A. M. 1996, ApJ, 470, 403 1997, ApJ, 474, 140 1999, ApJS, 121, 369 2000, ApJ, 533, L5 2002, ApJ, 566, 68 (PW02)

Prochaska, J. X., et al. 2001b, ApJS, 137, 21

Rao, S. M., \& Turnshek, D. A. 2000, ApJS, 130, 1

Rosenberg, J. L., \& Schneider, S. E. 2003, ApJ, 585, 256

Savaglio, S., Fall, S. M., \& Fiore, F. 2003, ApJ, 585, 638

Schaye, J., Aguirre, A., Kim, T.-S., Theuns, T., Rauch, M., \& Sargent, W. L. W. 2003, ApJ, in press (astro-ph/0306469)

Sheinis, A. I., Miller, J., Bigelow, B., Bolte, M., Epps, H., Kibrick, R., Radovan, M., \& Sutin, B. 2002, PASP, 114, 851

Somerville, R. S., Primack, J. R., \& Faber, S. M. 2001, MNRAS, 320, 504 Songaila, A. 2001, ApJ, 561, L153

Songaila, A., \& Cowie, L. L. 2002, AJ, 123, 2183

Srianand, R., Petitjean, P., \& Ledoux C. 2000, Nature, 408, 931

Storrie-Lombardi, L. J., \& Wolfe, A. M. 2000, ApJ, 543, 552

Tissera, P. B., Lambas, D. G., Mosconi, M. B., \& Cora, S. 2001, ApJ, 557, 527

Vladilo, G. 2002, A\&A, 391, 407

Vladilo, G., Bonifacio, P., Centurión, M., \& Molaro, P. 2000, ApJ, 543, 24

Wasserburg, G. J., \& Qian, Y.-Z. 2000, ApJ, 538, L99

Wolfe, A. M., Fan, X.-M., Tytler, D., Vogt, S. S., Keane, M. J., \& and Lanzetta, K. M. 1994, ApJ, 435, L101

Wolfe, A. M., Gawiser, E., \& Prochaska, J. X. 2003a, ApJ, in press

Wolfe, A. M., Lanzetta, K. M., Foltz, C. B., \& Chaffee, F. H. 1995, ApJ, 454, 698

Wolfe, A. M., Prochaska, J. X., \& Gawiser, E. 2003b, ApJ, in press 